\title{
A new approach to the cheap LQ regulator exploiting the geometric properties of the Hamiltonian system *
}

\author{
Domenico Prattichizzo $^{\text {a }}$, Lorenzo Ntogramatzidis ${ }^{\mathrm{b}}$, Giovanni Marro ${ }^{\mathrm{c}}$, \\ a Dipartimento di Ingegneria dell' Informazione, Università di Siena, via Roma 56 - I-53100, Italy. \\ ${ }^{\mathrm{b}}$ Department of Electrical and Electronic Engineering, The University of Melbourne, Parkville, VIC 3010, Australia \\ ${ }^{\mathrm{c}}$ Dipartimento di Elettronica, Informatica e Sistemistica, Università di Bologna, viale Risorgimento, 2 - 40136 Bologna, Italy
}

\begin{abstract}
The cheap LQ regulator is reinterpreted as an output nulling problem which is a basic problem of the geometric control theory. In fact, solving the LQ regulator problem is equivalent to keep the output of the related Hamiltonian system identically zero. The solution lies on a controlled invariant subspace whose dimension is characterized in terms of the minimal conditioned invariant of the original system, and the optimal feedback gain is computed as the friend matrix of the resolving subspace. This study yields a new computational framework for the cheap LQ regulator, relying only on the very basic and simple tools of the geometric approach, namely the algorithms for controlled and conditioned invariant subspaces and invariant zeros.
\end{abstract}

Key words: Geometric Techniques, Cheap LQ Problem, Hamiltonian System, Continuous-Time Systems.

\section{Introduction}

It is well known that the solution of the infinite-horizon linear quadratic (LQ) regulator problem strongly depends on the matrix weighting the input in the cost function, traditionally denoted by $R$. When $R$ is positive definite, the problem is said to be regular (see e.g. $[1,8]$ ). When $R$ is positive semidefinite, the problem is called singular, and cheap when $R$ is zero. The singular and cheap cases have been treated within the framework of geometric control theory, see for example $[6,19,13]$ and references therein. In particular, in [6] and [19] it was proved that an optimal solution of the singular problem exists for all initial conditions if the class of allowable controls is extended to include distributions. The approach taken in [13] is based on an analysis of the structure of both singular and cheap problems (where the latter is treated as the limiting case of the former)

\footnotetext{
* This paper was not presented at any IFAC meeting. Partially supported by the ministry of higher education of Italy (MIUR) under the project Identification and Control of Industrial Systems and by the Australian Research Council (DP0664789). Corresponding author D. Prattchizzo.

Email addresses: prattichizzo@ing.unisi.it (Domenico Prattichizzo), lnt@ee.unimelb.edu.au (Lorenzo Ntogramatzidis), gmarro@deis.unibo.it (Giovanni Marro).
}

carried out by means of the so-called special coordinate basis. Valuable results on this issue have also been obtained in $[14,17,16,15]$, where the solution of the singular LQ problems is based on linear matrix inequalities.

This paper proposes a new perspective for the solution of the cheap LQ regulator. The keystone is the interpretation of the LQ regulator as an output nulling problem referred to the Hamiltonian system. This new approach enables to solve the cheap LQ regulator by only using the basic and computationally efficient tools of the geometric approach, namely the algorithms for controlled and conditioned invariance and invariant zeros, $[2,4,18,20]$. By writing the conditions for optimality in the form of the Hamiltonian system, whose output has to be maintained identically equal to zero, the cheap LQ problem reduces to finding a state feedback such that the statecostate trajectory entirely lies on an internally stabilizable output nulling subspace of the Hamiltonian system. In the framework of the geometric approach, this is a standard unknown-input decoupling problem.

This work is intended to be the counterpart of [10] for the continuous time. The continuous time case is different from the discrete time one. In fact, differently from the discrete time, in the continuous time the optimal statefeedback does not exist for any initial condition, so that significant issues related to existence of optimal solutions 
arise. Solving the cheap LQ problem by directly referring to the Hamiltonian system provides a very simple characterization of the subspace of all admissible initial conditions such that a state-feedback solution exists.

Notation. The symbol $\mathbb{R}^{n \times m}$ is used to denote the space of $n \times m$ real constant matrices. The image and the nullspace of matrix $M \in \mathbb{R}^{n \times m}$ are respectively denoted by $\operatorname{im} M$ and $\operatorname{ker} M$. Given a subspace $\mathcal{Y}$ of $\mathbb{R}^{n}$, the symbol $M^{-1} \mathcal{Y}$ stands for the inverse image of $\mathcal{Y}$ with respect to the linear transformation $M$, while $\mathcal{Y}^{\perp}$ represents the orthogonal complement of $\mathcal{Y}$. Denote by $M^{T}$ and by $M^{\dagger}$ the transpose and the Moore-Penrose pseudo-inverse of $M$, respectively; since the identity $\left(M^{-1}\right)^{T}=\left(M^{T}\right)^{-1}$ holds, the symbol $M^{-T}$ is used concisely. The symbol $I_{d}$ will stand for the $d \times d$ identity matrix. Finally, the symbol $\mathbb{N}$ denotes the set of non-negative integer numbers.

\section{Statement of the Problem}

Consider the linear time-invariant system

$$
\begin{aligned}
& \dot{x}(t)=A x(t)+B u(t), \quad x(0)=x_{0}, \\
& y(t)=C x(t),
\end{aligned}
$$

where, for all $t \geq 0, x(t) \in \mathbb{R}^{n}$ is the state, $u(t) \in \mathbb{R}^{m}$ is the control input, $y(t) \in \mathbb{R}^{p}$ is the output, $A, B$ and $C$ are real constant matrices of proper sizes. With no loss of generality it is assumed that $B$ has linearly independent columns and $C$ has linearly independent rows. System (1) is briefly referred to as the triple $(A, B, C)$, and is concisely denoted by $\Sigma$. We make the following standing assumptions on $\Sigma$ :

(A1) the pair $(A, B)$ is stabilizable;

(A2) $\Sigma$ has no invariant zeros on the imaginary axis.

The cheap LQ problem considered in this paper can be formulated as follows.

Problem 2.1 Find the subspace $\mathcal{L}$ of initial conditions of $\Sigma$ for which a matrix $K \in \mathbb{R}^{m \times n}$ exists such that:

(1) the closed-loop matrix $A-B K$ is stable;

(2) for all $x_{0} \in \mathcal{L}$, the state trajectory generated by (1) with initial state $x(0)=x_{0}$ and with the input $u(t)=$ $-K x(t)$ minimizes the quadratic cost function

$$
J=\frac{1}{2} \int_{0}^{\infty} y^{T}(t) y(t) d t=\frac{1}{2} \int_{0}^{\infty} x^{T}(t) C^{T} C x(t) d t
$$

For $x_{0} \in \mathcal{L}$, determine $K$ such that (i)-(ii) are satisfied.

Problem 2.1 is usually referred to as a cheap LQ problem, since the input function is not explicitly weighted in the performance index $J$. Differently from other approaches, here the optimal control is sought within the class of static state feedback inputs, so that optimal solutions containing distributions are ruled out. As will become clear in Section 4, this poses a problem of existence of optimal solutions to Problem 2.1. When Problem 2.1 admits solutions, the optimal feedback matrix does not depend on the particular initial state in $\mathcal{L}$. This issue is discussed in Section 5.

\section{Geometric Background}

The geometric setting developed here requires the following notations: $\mathcal{V}_{\Sigma}^{*}$ stands for the largest $(A, \operatorname{im} B)$ controlled invariant subspace of $(A, B, C)$ contained in ker $C$, which is also denoted by $\max \mathcal{V}(A, \operatorname{im} B, \operatorname{ker} C)$. Recall that $\mathcal{V}_{\Sigma}^{*}$ is the subspace of all initial states $x_{0} \in \mathbb{R}^{n}$ of (1) for which an input exists such that the corresponding output is identically zero, and can be computed as the last term of the monotonically non-increasing sequence of subspaces $\left(\mathcal{V}_{i}\right)_{i \in \mathbb{N}}$ described by $\mathcal{V}_{0}=\operatorname{ker} C, \mathcal{V}_{i}=\operatorname{ker} C \cap A^{-1}\left(\mathcal{V}_{i-1}+\operatorname{im} B\right)$, $i=1, \ldots, k$, where the integer $k \leq n-1$ is determined by the condition $\mathcal{V}_{i+1}=\mathcal{V}_{i}\left(=\mathcal{V}_{\Sigma}^{*}\right)$, see e.g. [4, p.210]. The symbol $\mathcal{S}_{\Sigma}^{*}$ stands for the smallest $(A, \operatorname{ker} C)$ conditioned invariant subspace containing im $B$, which is denoted by $\min \mathcal{S}(A, \operatorname{ker} C, \operatorname{im} B)$, and can be computed as the last term of the monotonically nondecreasing sequence $\left(\mathcal{S}_{i}\right)_{i \in \mathbb{N}}$ described by $\mathcal{S}_{0}=\operatorname{im} B$, $\mathcal{S}_{i}=\operatorname{im} B+A\left(\mathcal{S}_{i-1} \cap \operatorname{ker} C\right), i=1, \ldots, k$, where now the integer $k \leq n-1$ is determined by the condition $\mathcal{S}_{i+1}=\mathcal{S}_{i}\left(=\mathcal{S}_{\Sigma}^{*}\right)$, see [4, p.209]. The notion and related geometric properties of left and right-invertibility are now briefly recalled, see [4, p.237] and [18, p.189] for details.

Property 3.1 The following statements are equivalent:

(1) $\Sigma$ is left-invertible;

(2) $\mathcal{V}_{\Sigma}^{*} \cap \mathcal{S}_{\Sigma}^{*}=\{0\}$;

(3) $B^{-1} \mathcal{V}_{\Sigma}^{*}=\{0\}$

Roughly speaking, if $\Sigma$ is not left-invertible, the input function corresponding to a given response (obtained with zero initial condition) can only be determined modulo $B^{-1} \mathcal{V}_{\Sigma}^{*} \subseteq \mathbb{R}^{m}$, see [3, Theorem 3]. The subspace $B^{-1} \mathcal{V}_{\Sigma}^{*}$ is therefore called input functional unobservability subspace. Dually, the following property holds.

Property 3.2 The following statements are equivalent:

(1) $\Sigma$ is right-invertible;

(2) $\mathcal{V}_{\Sigma}^{*}+\mathcal{S}_{\Sigma}^{*}=\mathbb{R}^{n}$;

(3) $C \mathcal{S}_{\Sigma}^{*}=\mathbb{R}^{p}$.

If $\Sigma$ is not right-invertible, the output function can be imposed modulo any complement of the subspace $C \mathcal{S}_{\Sigma}^{*} \subseteq \mathbb{R}^{p}$, see $\left[3\right.$, Theorem 4]. Thus, the subspace $C \mathcal{S}_{\Sigma}^{*}$ 
is called output functional reachability subspace.

Denote by $\Sigma^{T}$ the dual of system $\Sigma$, described by the triple $\left(A^{T}, C^{T}, B^{T}\right)$.

Property 3.3 System $\Sigma$ is left (right) invertible if and only if its dual $\Sigma^{T}$ is right (left) invertible.

The proof of the former follows from the well known identities $\mathcal{V}_{\Sigma}^{*}=\left(\mathcal{S}_{\Sigma^{T}}^{*}\right)^{\perp}$ and $\mathcal{S}_{\Sigma}^{*}=\left(\mathcal{V}_{\Sigma T}^{*}\right)^{\perp}$, see e.g. [4, p.209]. Recall that, given a friend $F$ of $\mathcal{V}_{\Sigma}^{*}$, i.e. a matrix such that $(A+B F) \mathcal{V}_{\Sigma}^{*} \subseteq \mathcal{V}_{\Sigma}^{*}$, the eigenvalues of $A+B F$ restricted to $\mathcal{V}_{\Sigma}^{*}$ are split into two sets. The eigenvalues of $A+B F$ which are restricted to $\mathcal{V}_{\Sigma}^{*} \cap \mathcal{S}_{\Sigma}^{*}$ are all assignable by a suitable choice of $F$. The eigenvalues of $A+B F$ restricted to the quotient $\mathcal{V}_{\Sigma}^{*} / \mathcal{V}_{\Sigma}^{*} \cap \mathcal{S}_{\Sigma}^{*}$ are fixed for any friend of $\mathcal{V}_{\Sigma}^{*}$, and are called invariant zeros of $\Sigma$. If all the invariant zeros of $\Sigma$ have strictly negative real part, $\Sigma$ is said to be minimum phase and $\mathcal{V}_{\Sigma}^{*}$ is said to be internally stabilizable. The subspace $\mathcal{R}_{\Sigma}^{*}:=\mathcal{V}_{\Sigma}^{*} \cap \mathcal{S}_{\Sigma}^{*}$ is often referred to as the reachable subspace on $\mathcal{V}_{\Sigma}^{*}$, see [11]. By virtue of Property 3.1, if $\Sigma$ is left-invertible, the set of invariant zeros reduces to the set of eigenvalues of $A+B F$ restricted to $\mathcal{V}_{\Sigma}^{*}$. For a detailed analysis of the internal and external eigenstructure of a controlled invariant subspace we refer to $[4$, pp.217-222] and [18, pp.89-96].

Notice that the dynamical system whose evolution is described by (1) can be written backwards in time as

$$
\begin{aligned}
& \dot{z}(t)=-A z(t)-B u(t), \\
& y(t)=C z(t) .
\end{aligned}
$$

The triple $(-A,-B, C)$, here denoted by $\Sigma^{-1}$, is therefore referred to as the time-reversed system associated with $\Sigma$. Note that

$$
\mathcal{V}_{\Sigma}^{*}=\mathcal{V}_{\Sigma^{-1}}^{*} \quad \text { and } \quad \mathcal{S}_{\Sigma}^{*}=\mathcal{S}_{\Sigma^{-1}}^{*}
$$

since the definitions of $\mathcal{V}_{\Sigma}^{*}$ and $\mathcal{S}_{\Sigma}^{*}$ do not depend on the sign of the matrices $A$ and $B$. Hence, the following property holds.

Property 3.4 System $\Sigma$ is left (right) invertible if and only if $\Sigma^{-1}$ is left (right) invertible.

\section{A Geometric Insight into the Structure of the Hamiltonian System}

Recall that the optimal state trajectory and control law for Problem 2.1 satisfy the following equations

$$
\begin{aligned}
& \dot{x}(t)=A x(t)+B u(t), \\
& \dot{\lambda}(t)=-C^{T} C x(t)-A^{T} \lambda(t), \\
& B^{T} \lambda(t)=0,
\end{aligned}
$$

obtained by extending the state $x(t)$ of system (1) with the costate function $\lambda(t) \in \mathbb{R}^{n}(t \geq 0)$. Equations (3-5) can be obtained from the computation of the derivatives of the Hamiltonian function with respect to $x(t), \lambda(t)$ and $u(t)$, see e.g. [8, pp.131-133].

A fundamental observation for the approach taken here is that (3-5) can be written in the compact form:

$$
\begin{aligned}
{\left[\begin{array}{l}
\dot{x}(t) \\
\dot{\lambda}(t)
\end{array}\right] } & =\left[\begin{array}{cc}
A & 0 \\
-C^{T} C-A^{T}
\end{array}\right]\left[\begin{array}{l}
x(t) \\
\lambda(t)
\end{array}\right]+\left[\begin{array}{l}
B \\
0
\end{array}\right] u(t) \\
\hat{y}(t) & =\left[\begin{array}{cc}
0 & B^{T}
\end{array}\right]\left[\begin{array}{l}
x(t) \\
\lambda(t)
\end{array}\right]=0,
\end{aligned}
$$

and that (6) can be regarded as a $2 n$ order system whose output is identically zero. The matrices in (6) are respectively denoted by the symbols $\widehat{A}, \widehat{B}$ and $\widehat{C}$, while the triple $(\widehat{A}, \widehat{B}, \widehat{C})$ is denoted by $\widehat{\Sigma}$, and is referred to as the Hamiltonian system. From the structure of the Hamiltonian system, which along with the boundary equations represents a set of necessary and sufficient conditions for optimality, it is clear that Problem 2.1 can be reformulated as the problem of finding the control law maintaining $\hat{y}(t)=0$ for all $t \geq 0$ for the assigned initial condition $x(0)=x_{0}$, and such that the corresponding state trajectory converges to the origin as $t$ approaches infinity. Clearly, this aim can be achieved if and only if the initial condition $x_{0}$ is such that an initial value of the costate $\lambda_{0}:=\lambda(0)$ exists so that $\left[\begin{array}{l}x_{0} \\ \lambda_{0}\end{array}\right]$ belongs to an internally stabilizable $(\widehat{A}, \operatorname{im} \widehat{B})$-controlled invariant contained in the null-space of $\widehat{C}$.

The following lemma provides an important characterization of the geometric structure of the Hamiltonian system in terms of left and right-invertibility. The further assumption of left-invertibility of $\Sigma$ is technical in nature and will be removed in Section 5.1.

Lemma 4.1 If $\Sigma$ is left-invertible, the Hamiltonian system $\widehat{\Sigma}$ is both left and right-invertible.

Proof: First, note that $\widehat{\Sigma}$ is the series connection of $\Sigma$ and the time-reversed representation of the dual of $\Sigma$, henceforth denoted by the symbol $\Sigma^{-T}$, and representing the triple $\left(-A^{T},-C^{T}, B^{T}\right)$. As a consequence of Properties 3.3 and 3.4, it follows that $\Sigma^{-T}$ is rightinvertible. The block-structure of the Hamiltonian system matches that described in [10], even if the inner structures of $\Sigma$ and $\Sigma^{-T}$ in the continuous and discrete cases are different. Hence, the proof of the rightinvertibility of $\widehat{\Sigma}$ can be carried out by employing the same arguments used in [10, Lemma 1], which are based on the fact that the input reachability subspace of $\Sigma$ and 
the orthogonal complement of the input unobservability subspace of $\Sigma^{-T}$ are equal ${ }^{1}$. Finally, a simple computation shows that the time-reversed representation of the Hamiltonian system $\widehat{\Sigma}^{-1}$ and its dual $\widehat{\Sigma}^{T}$ are linearly equivalent, i.e., the triple $(-\widehat{A},-\widehat{B}, \widehat{C})$ is obtained from the triple $\left(\widehat{A}^{T}, \widehat{C}^{T}, \widehat{B}^{T}\right)$ via a change of coordinates in $\mathbb{R}^{2 n}$ given by the matrix $T=\left[\begin{array}{cc}0 & I_{n} \\ -I_{n} & 0\end{array}\right]$. As a result, $\widehat{\Sigma}^{-1}$ and $\widehat{\Sigma}$ are left-invertible, since such is $\widehat{\Sigma}^{T}$.

In the following, the symbols $\mathcal{V}_{\widehat{\Sigma}}^{*}:=\max \mathcal{V}(\widehat{A}, \operatorname{im} \widehat{B}, \operatorname{ker} \widehat{C})$ and $\mathcal{S}_{\widehat{\Sigma}}^{*}:=\min \mathcal{S}(\widehat{A}, \operatorname{ker} \widehat{C}, \operatorname{im} \widehat{B})$ will be used, consistently with the notation previously introduced.

Corollary 4.1 Let $\Sigma$ be left-invertible. The set of invariant zeros of the Hamiltonian system $\widehat{\Sigma}$ is mirrored with respect to the imaginary axis, and is devoid of zeros on the imaginary axis.

Proof: First, let us show that the invariant zeros of $\widehat{\Sigma}$ are in pairs $(z,-z)$. Let $z$ be an invariant zero of $\widehat{\Sigma}$, i.e., it is an eigenvalue of $(\widehat{A}+\widehat{B} \widehat{F})$ restricted to $\mathcal{V}_{\widehat{\Sigma}}^{*}$, where $\widehat{F}$ is a friend of $\mathcal{V}_{\widehat{\Sigma}}^{*}$, since, owing to Lemma $4.1, \widehat{\Sigma}$ is leftinvertible. It follows that $z$ is an invariant zero of $\widehat{\Sigma}^{T}$, hence it is also an invariant zero of $\widehat{\Sigma}^{-1}$, since in the proof of Lemma 4.1 it was shown that $\widehat{\Sigma}^{T}$ and $\widehat{\Sigma}^{-1}$ are linearly equivalent up to a change of basis. However, since owing to $(2) \mathcal{V}_{\widehat{\Sigma}}^{*}=\mathcal{V}_{\widehat{\Sigma}-1}^{*}$ and the eigenvalues of $(\widehat{A}+\widehat{B} \widehat{F})$ and those of $(-\widehat{A}-\widehat{B} \widehat{F})$ are opposite, it is found that $-z$ is an invariant zero of $\widehat{\Sigma}$. Now we show that $\widehat{\Sigma}$ has no invariant zeros on the imaginary axis. Suppose by contradiction that $z_{0}=j \omega_{0}$ is a zero of $\widehat{\Sigma}$; by definition of zero, it follows that for a suitable initial condition $\left[\begin{array}{c}x_{0} \\ \lambda_{0}\end{array}\right]$ of $\widehat{\Sigma}$, the input $u(t)=e^{z_{0} t}=e^{j \omega_{0} t}$ gives rise to an indentically zero output $\hat{y}$, which implies that the state trajectory is optimal for a cheap problem with $x(0)=x_{0}$, [9]. However, since $z_{0}$ cannot be a zero of $\Sigma$ in view of Assumption (A2), the same control $u(t)=e^{z_{0} t}=e^{j \omega_{0} t}$ gives rise to a non-zero output of the system $\Sigma$. More precisely, $y(t)=y_{0} e^{j \omega_{0} t}$ for a suitable $y_{0} \in \mathbb{R}^{p} \backslash\{0\}$, $\left[21\right.$, Lemma 3.36]. When $\omega_{0}=0$, this output is constant. When $\omega_{0} \neq 0$, since $-z_{0}$ is a zero of the Hamiltonian system as well in view of the first part of the proof, then an input $u(t)=\sin \left(\omega_{0} t\right)$ gives rise to the output $y(t)=y_{0} \sin \left(\omega_{0} t+\varphi_{0}\right)$. In both cases, $\int_{0}^{\infty} y^{T}(t) y(t) d t$ is not finite. On the other hand, a finite cost can always be achieved in view of Assumption (A1), so that this solution would not be optimal.

Corollary 4.2 Let $\Sigma$ be left-invertible. The internal eigenvalues of $\mathcal{V}_{\widehat{\Sigma}}^{*}$ are the invariant zeros of the Hamil-

1 Recall that if $M \in \mathbb{R}^{n \times m}$ is a and $\mathcal{Y}$ is a subspace of $\mathbb{R}^{n}$, there holds $M^{T} \mathcal{Y}^{\perp}=\left(M^{-1} \mathcal{Y}\right)^{\perp},[4$, Property 3.1-3, p.128]. tonian system $\widehat{\Sigma}$. These are in pairs of the type $(z,-z)$. This set of zeros includes the invariant zeros of $\Sigma$.

Proof: The proof follows from Lemma 4.1, which ensures that $\mathcal{V}_{\widehat{\Sigma}}^{*}$ has no arbitrarily assignable poles, and from Corollary 4.1. The fact that the set of zeros of $\widehat{\Sigma}$ includes the invariant zeros of $\Sigma$ comes from the fact that $\widehat{\Sigma}$ is the series connection of $\Sigma$ and $\Sigma^{-T}$.

Now we define a new system $\bar{\Sigma}$ that plays a key role in providing a characterization of the geometric structure of the Hamiltonian system. Consider a basis matrix $U$ of the output functional reachability subspace $C \mathcal{S}_{\Sigma}^{*} \subseteq \mathbb{R}^{p}$ of $\Sigma$. Let the symbol $\bar{\Sigma}$ represent the system associated with the triple $\left(A^{T}, C^{T} U, B^{T}\right)$. System $\bar{\Sigma}$ is similar in structure to the dual system $\Sigma^{T}$, but is such that its input actions are restricted to belonging to the output functional reachability subspace of $\Sigma$. Moreover, differently from $\Sigma^{T}$, which is only right-invertible, now it is shown that $\bar{\Sigma}$ is both left and right-invertible.

Lemma 4.2 System $\bar{\Sigma}$ is left and right-invertible, and $\mathcal{V}_{\Sigma}^{*}=\mathcal{V}_{\Sigma-T}^{*}$.

Proof: The left-invertibility can be proved as follows. Let $\xi$ be a non-null vector of $\left(C^{T} U\right)^{-1} \mathcal{V}_{\Sigma}^{*}$ where $\quad \mathcal{V}_{\bar{\Sigma}}^{*}:=\max \mathcal{V}\left(A^{T}, C^{T} C \mathcal{S}_{\Sigma}^{*}\right.$, ker $\left.B^{T}\right)$. Hence, $C^{T} U \xi \in \mathcal{V}_{\bar{\Sigma}}^{*}$. As a consequence, a non-null vector $\xi^{\prime}:=U \xi$ exists such that $\xi^{\prime} \in C^{-T} \mathcal{V}_{\Sigma}^{*}=\left(C \mathcal{S}_{\Sigma}^{*}\right)^{\perp}$. However, since $\xi^{\prime}$ lies in the range of $U$ as well, it follows that $\xi^{\prime}=0$, so that $\xi=0$ since $\operatorname{ker} U=\{0\}$, and, owing to Property 3.1, $\bar{\Sigma}$ is left-invertible. Now, we show that $\mathcal{V}_{\Sigma}^{*}=\mathcal{V}_{\Sigma-T}^{*}$. Since the set of control actions of $\bar{\Sigma}$ is restricted with respect to that of $\Sigma^{T}$, by definition the set of output-nulling subspaces of $\bar{\Sigma}$ contains the set of output-nulling suspaces of $\Sigma^{T}$. Hence, in particular, $\mathcal{V}_{\bar{\Sigma}}^{*} \subseteq \mathcal{V}_{\Sigma^{T}}^{*}$. We only need to prove that the opposite inclusion holds as well, i.e., $\mathcal{V}_{\bar{\Sigma}}^{*} \supseteq \mathcal{V}_{\Sigma^{T}}^{*}$. To this end, it suffices to show that $\mathcal{V}_{\Sigma^{T}}^{*}$ is output-nulling of $\bar{\Sigma}$. Consider the identity $\operatorname{im} C^{T}=C^{T} \mathbb{R}^{p}=C^{T}\left(\left(C^{-T} \mathcal{V}_{\Sigma^{T}}^{*}\right) \oplus\left(C^{-T} \mathcal{V}_{\Sigma^{T}}^{*}\right)^{\perp}\right)=$ $\left(\mathcal{V}_{\Sigma^{T}}^{*} \cap \operatorname{im} C^{T}\right)+C^{T}\left(C^{-T} \mathcal{V}_{\Sigma^{T}}^{*}\right)^{\perp}$. By adding $\mathcal{V}_{\Sigma^{T}}^{*}$ to both sides of the latter it easily follows that $\mathcal{V}_{\Sigma^{T}}^{*}+\operatorname{im} C^{T}=$ $\mathcal{V}_{\Sigma^{T}}^{*}+C^{T}\left(C^{-T} \mathcal{V}_{\Sigma^{T}}^{*}\right)^{\perp}=\mathcal{V}_{\Sigma^{T}}^{*}+C^{T} C \mathcal{S}_{\Sigma}^{*}$ and, since $A^{T} \mathcal{V}_{\Sigma^{T}}^{*} \subseteq \mathcal{V}_{\Sigma^{T}}^{*}+\operatorname{im} C^{T}$ as $\mathcal{V}_{\Sigma^{T}}^{*}$ is controlled invariant for $\Sigma^{T}$, it also follows that $A^{T} \mathcal{V}_{\Sigma^{T}}^{*} \subseteq \mathcal{V}_{\Sigma^{T}}^{*}+C^{T} C \mathcal{S}_{\Sigma}^{*}$. This means that $\mathcal{V}_{\Sigma^{T}}^{*}$ is controlled invariant for $\bar{\Sigma}$, as well. As a result, $\mathcal{V}_{\Sigma}^{*}=\mathcal{V}_{\Sigma^{T}}^{*}$, so that $\mathcal{V}_{\Sigma}^{*}=\mathcal{V}_{\Sigma^{-T}}^{*}$ by $(2)$. Now, denoting by $\left(\mathcal{V}_{i}\right)_{i \in \mathbb{N}}$ and by $\left(\overline{\mathcal{V}}_{i}\right)_{i \in \mathbb{N}}$ the sequences of subspaces $\left(\mathcal{V}_{i}\right)_{i \in \mathbb{N}}$ referred to $\Sigma^{-T}$ and $\bar{\Sigma}$, respectively, we show by induction that $\mathcal{V}_{i}=\overline{\mathcal{V}}_{i}$ for all $i \leq n-1$. The identity holds for $i=0$ since $\mathcal{V}_{0}=\overline{\mathcal{V}}_{0}=\operatorname{ker} B^{T}$. Suppose $\mathcal{V}_{k}=\overline{\mathcal{V}}_{k}$ for any $k>0$. Since the sequence $\left(\mathcal{V}_{i}\right)_{i \in \mathbf{N}}$ is monotonically non-decreasing, $\overline{\mathcal{V}}_{k} \supseteq \mathcal{V}_{\bar{\Sigma}}^{*}=\mathcal{V}_{\Sigma^{T}}^{*} \supseteq$ $\mathcal{V}_{\Sigma}^{*} \cap \operatorname{im} C^{T}=\left(\mathcal{S}_{\Sigma}^{*}\right)^{\perp} \cap \operatorname{im} C^{T}=C^{T}\left(C \mathcal{S}_{\Sigma}^{*}\right)^{\perp}$. Hence, it 
is now found that $\mathcal{V}_{k+1}=\operatorname{ker} B^{T} \cap A^{-T}\left(\mathcal{V}_{k}+\operatorname{im} C^{T}\right)=$ $\operatorname{ker} B^{T} \cap A^{-T}\left(\overline{\mathcal{V}}_{k}+C^{T} C \mathcal{S}_{\Sigma}^{*}+C^{T}\left(C \mathcal{S}_{\Sigma}^{*}\right)^{\perp}\right)=\operatorname{ker} B^{T} \cap$ $A^{-T}\left(\overline{\mathcal{V}}_{k}+C^{T} C \mathcal{S}_{\Sigma}^{*}\right)=\overline{\mathcal{V}}_{k+1}$, since $\overline{\mathcal{V}}_{k} \supseteq C^{T}\left(C \mathcal{S}_{\Sigma}^{*}\right)^{\perp}$. Finally, to prove that $\bar{\Sigma}$ is right-invertible, we show by induction that $\mathcal{S}_{i}+\mathcal{V}_{i}=\overline{\mathcal{S}}_{i}+\overline{\mathcal{V}}_{i}$ for all $i \leq n-1$. Since $\mathcal{S}_{\Sigma}^{*}$ contains im $B$, it is found that $C^{T}\left(C \mathcal{S}_{\Sigma}^{*}\right)^{\perp} \subseteq \operatorname{ker} B^{T}$. Thus, $\mathcal{S}_{0}+\mathcal{V}_{0}=\operatorname{im} C^{T}+\operatorname{ker} B^{T}=C^{T}\left(C \mathcal{S}_{\Sigma}^{*}\right)+$ $C^{T}\left(C \mathcal{S}_{\Sigma}^{*}\right)^{\perp}+\operatorname{ker} B^{T}=C^{T}\left(C \mathcal{S}_{\Sigma}^{*}\right)+\operatorname{ker} B^{T}=\overline{\mathcal{S}}_{0}+\overline{\mathcal{V}}_{0}$. Let now $\mathcal{S}_{k}+\mathcal{V}_{k}=\overline{\mathcal{S}}_{k}+\overline{\mathcal{V}}_{k}$ for a given $k>0$. Consider the trivial identity $\operatorname{ker} B^{T}=\left(\mathcal{S}_{k} \cap \operatorname{ker} B^{T}\right)+$ $\operatorname{ker} B^{T}=\left(\overline{\mathcal{S}}_{k} \cap \operatorname{ker} B^{T}\right)+\operatorname{ker} B^{T}$. By applying $A^{T}$ to the latter, by the set of inclusions $A^{T} \operatorname{ker} B^{T} \subseteq$ $A^{T}\left(\operatorname{ker} B^{T}+\operatorname{im} C^{T}\right) \subseteq \mathcal{V}_{1} \subseteq \mathcal{V}_{k+1}$ for all $k \geq 1$, and since as already observed $\mathcal{V}_{k+1}=\overline{\mathcal{V}}_{k+1}$ and $\mathcal{V}_{i}=\overline{\mathcal{V}}_{i} \supseteq C^{T}\left(C \mathcal{S}_{\Sigma}^{*}\right)^{\perp}$ for all $i \geq 0$, it follows that $\mathcal{S}_{k+1}+\mathcal{V}_{k+1}=\operatorname{im} C^{T}+A^{T}\left(\mathcal{S}_{k} \cap \operatorname{ker} B^{T}\right)+\mathcal{V}_{k+1}=$ $C^{T} C \mathcal{S}_{\Sigma}^{*}+A^{T}\left(\mathcal{S}_{k} \cap \operatorname{ker} B^{T}\right)+\mathcal{V}_{k+1}=\overline{\mathcal{S}}_{k+1}+\overline{\mathcal{V}}_{k+1}$. In particular, it follows that $\bar{\Sigma}$ is right-invertible since such is $\Sigma$.

Let $r$ be the dimension of $\mathcal{S}_{\Sigma}^{*}$. As a consequence of Lemma 4.2, we get $\operatorname{dim} \mathcal{V}_{\Sigma}^{*}=\operatorname{dim} \mathcal{V}_{\Sigma^{-T}}^{*}=\operatorname{dim} \mathcal{V}_{\Sigma^{T}}^{*}=$ $\operatorname{dim}\left(\mathcal{S}_{\Sigma}^{*}\right)^{\perp}=n-r$. However, since $\mathcal{V}_{\bar{\Sigma}}^{*} \oplus \mathcal{S}_{\bar{\Sigma}}^{*}=\mathbb{R}^{n}$, it follows that

$$
\operatorname{dim} \mathcal{S}_{\bar{\Sigma}}^{*}=n-\operatorname{dim} \mathcal{V}_{\Sigma}^{*}=r .
$$

The following fundamental result relates the structure of the previously defined system $\bar{\Sigma}$ with that of the Hamiltonian system $\widehat{\Sigma}$. In particular, the structure and the dimension of the smallest conditioned invariant subspace of $\widehat{\Sigma}$ containing the image of $\widehat{B}$ is expressed as a function of the structure and dimension of the smallest conditioned invariants of $\Sigma$ and $\bar{\Sigma}$.

Lemma 4.3 Let $\Sigma$ be left-invertible, and let $r$ be the dimension of $\mathcal{S}_{\Sigma}^{*}$. Hence, the following equalities hold:

$\operatorname{dim} \mathcal{S}_{\widehat{\Sigma}}^{*}=2 r, \quad$ and $\quad \operatorname{dim} \mathcal{V}_{\widehat{\Sigma}}^{*}=2(n-r)$.

Proof: Denote by $S$ and by $\bar{S}$ two basis matrices for $\mathcal{S}_{\Sigma}^{*}$ and $\mathcal{S}_{\Sigma}^{*}$, respectively. The smallest conditioned invariant subspace of the Hamiltonian system $\mathcal{S}_{\widehat{\Sigma}}^{*}$ can be expressed as $\mathcal{S}_{\widehat{\Sigma}}^{*}=\operatorname{im}\left[\begin{array}{ll}S & \times \\ 0 & \bar{S}\end{array}\right]$. In order to prove this fact, recall that given a zero initial condition $x(0)=0$, the generic subspace $\mathcal{S}_{i}$ of the sequence $\left(\mathcal{S}_{i}\right)_{i \in \mathbb{N}}$ can be interpreted as the set of values that can be assigned through a control function $u(t)$ to $x^{(i)}\left(0^{+}\right)$, the $i$-th derivative of the state, while yielding $y^{(k)}\left(0^{+}\right)=0$ for all $k \leq i-1$. This interpretation of the subspaces $\mathcal{S}_{i}$ is the continuous counterpart of that given by Lemma 3 in [10] in the discrete case. Now we prove that $\operatorname{im}\left[\begin{array}{ll}S & \times \\ 0 & \bar{S}\end{array}\right]$ is contained in $\mathcal{S}_{\widehat{\Sigma}}^{*}$. First, we show that $\operatorname{im}\left[\begin{array}{l}S \\ 0\end{array}\right] \subseteq \mathcal{S}_{\widehat{\Sigma}}^{*}$.
Let $\tilde{x} \in \mathcal{S}_{\Sigma}^{*}$. The function $u(t)$ ensuring $x^{(n)}\left(0^{+}\right)=\tilde{x}$ and $y^{(k)}=0$ for all $k \leq n-1$ is such that $\lambda^{(k+1)}\left(0^{+}\right)=0$ and $\hat{y}^{(k)}\left(0^{+}\right)=0$ for all $k \leq n-1$. In particular $\lambda^{(n)}\left(0^{+}\right)=0$. If $\left(\widehat{\mathcal{S}}_{i}\right)_{i \in \mathbb{N}}$ is the sequence of subspaces in the extended state-space of the Hamiltonian system converging to $\mathcal{S}_{\widehat{\Sigma}}^{*}$, it follows that $\left[\begin{array}{l}\tilde{x} \\ 0\end{array}\right] \in \widehat{\mathcal{S}}_{n} \subseteq \widehat{\mathcal{S}}_{2 n}=\mathcal{S}_{\widehat{\Sigma}}^{*}$. Now, we prove that a matrix $L$ exists such that $\operatorname{im}\left[\begin{array}{c}L \\ \bar{S}\end{array}\right] \subseteq \mathcal{S}_{\widehat{\Sigma}}^{*}$. Let $\tilde{\lambda} \in \mathcal{S}_{\bar{\Sigma}}^{*}$. Hence, a $y(t)$ on $C \mathcal{S}_{\Sigma}^{*}$ exists such that $\lambda^{(n)}\left(0^{+}\right)=\tilde{\lambda}$ with $\hat{y}^{(k)}\left(0^{+}\right)=0$ for all $k \leq n-1$. It follows that a $u(t)$ steering the output of $\Sigma$ to $y(t)$ exists as $C \mathcal{S}_{\Sigma}^{*}$ is the output reachability subspace of $\Sigma$ and since the geometric condition for the perfect decoupling $C^{T}\left(C \mathcal{S}_{\Sigma}^{*}\right)^{\perp} \subseteq$ $\mathcal{V}_{\Sigma}^{*}=\mathcal{V}_{\Sigma^{-T}}^{*}$ holds $[4, \mathrm{p} .224]$. The value $x^{(n)}\left(0^{+}\right)=\tilde{x}$ determined by $u(t)$ is therefore such that $\left[\begin{array}{l}\tilde{x} \\ \tilde{\lambda}\end{array}\right] \in \operatorname{im}\left[\begin{array}{l}L \\ \bar{S}\end{array}\right] \subseteq$ $\mathcal{S}_{\widehat{\Sigma}}^{*}$. To prove the opposite inclusion, consider $\left[\begin{array}{c}\tilde{x} \\ \tilde{\lambda}\end{array}\right] \in \mathcal{S}_{\widehat{\Sigma}}^{*}$. First let $\tilde{\lambda}=0$. If $\tilde{x}$ does not belong to $\mathcal{S}_{\Sigma}^{*}$ by contradiction, $y^{(k)}\left(0^{+}\right) \neq 0$ for some $k \leq 2 n-1$ gives rise to $\lambda^{(2 n)}\left(0^{+}\right) \neq \tilde{\lambda}=0$ since $\operatorname{ker} C=\operatorname{ker} C^{T} C$. Hence, $\mathcal{S}_{\widehat{\Sigma}}^{*} \cap$ $\operatorname{im}\left[\begin{array}{c}I_{n} \\ 0\end{array}\right]=\operatorname{im}\left[\begin{array}{l}S \\ 0\end{array}\right]$. If $\tilde{\lambda} \neq 0$, a sufficient condition for $\hat{y}^{(k)}\left(0^{+}\right)=0$ with $k \leq 2 n-1$ would be $\tilde{\lambda} \in \mathcal{S}_{\Sigma^{-T}}^{*}$. However, since $\Sigma$ is not right-invertible, the control actions applied to $\Sigma^{-T}$ can only be imposed on $C \mathcal{S}_{\Sigma}^{*}$.Hence, $\tilde{\lambda} \in \mathcal{S}_{\bar{\Sigma}}^{*}$ implies that a $\tilde{x} \in \mathbb{R}^{n}$ exists such that $\left[\begin{array}{c}\tilde{x} \\ \tilde{\lambda}\end{array}\right] \in \mathcal{S}_{\widehat{\Sigma}}^{*}$. As a result of $(7)$, the dimension of $\mathcal{S}_{\widehat{\Sigma}}^{*}$ is $2 r$, yielding the first of (8). The second of (8) is a simple consequence of the left and right-invertibility of $\widehat{\Sigma}$.

Corollary 4.3 Let $\Sigma$ be both left and right-invertible. Then $\operatorname{dim} \mathcal{V}_{\Sigma}^{*}=2 \operatorname{dim} \mathcal{V}_{\Sigma}^{*}$.

Proof: The identity follows directly by the left and right-invertibility of $\Sigma$.

\section{Geometric Solution of the Cheap LQ Problem}

The following theorem provides a clear characterization of the resolvent controlled invariant subspace of the Hamiltonian system.

Theorem 5.1 If $\Sigma$ is left-invertible, an $(n-r)$ dimensional internally stabilizable $(\widehat{A}, i m \widehat{B})$-controlled invariant subspace $\widehat{\mathcal{V}}_{R}$ contained in ker $\widehat{C}$ exists such that all its $n-r$ poles, all unassignable, are strictly stable.

Proof: Since $\Sigma$ is supposed to be left-invertible, Lemma 4.1 ensures that $\mathcal{V}_{\widehat{\Sigma}}^{*}$ has no assignable poles. Moreover, from Corollary 4.1 it follows that the poles of $\mathcal{V}_{\widehat{\Sigma}}^{*}$, which are the invariant zeros of $\widehat{\Sigma}$, are pairs of the type $(z,-z)$, hence all stable-antistable owing to Assumption (A2). This set of zeros includes 
the invariant zeros of $\Sigma$. Since $\widehat{\Sigma}$ is left and rightinvertible, the matrix $T_{1}:=\left[T^{\prime} T^{\prime \prime}\right]$, where im $T^{\prime}=\mathcal{V}_{\widehat{\Sigma}}^{*}$ and $\operatorname{im} T^{\prime \prime}=\mathcal{S}_{\widehat{\Sigma}}^{*}$, is a basis matrix of $\mathbb{R}^{2 n}$. Since $\mathcal{V}_{\widehat{\Sigma}}^{*}$ is an $(\widehat{A}, \mathrm{im} \widehat{B})$-controlled invariant subspace, a matrix $\widehat{F} \in \mathbb{R}^{m \times 2 n}$ exists such that $\mathcal{V}_{\widehat{\Sigma}}^{*}$ is an $(\widehat{A}+\widehat{B} \widehat{F})$ invariant. By performing the coordinate transformation defined by $T_{1}$, one obtains the following partitioned structure $\widehat{A}_{F, T_{1}}:=T_{1}^{-1}(\widehat{A}+\widehat{B} \widehat{F}) T_{1}=\left[\begin{array}{cc}M_{S U} \times \\ 0 & \times\end{array}\right]$, where the eigenvalues of $M_{S U} \in \mathbb{R}^{2(n-r) \times 2(n-r)}$ are the poles of $\mathcal{V}_{\widehat{\Sigma}}^{*}$, all unassignable by virtue of the leftinvertibility of $\widehat{\Sigma}$, are the strictly stable invariant zeros of the Hamiltonian system and their opposite. Since the subspace of the internal modes of $\mathcal{V}_{\widehat{\Sigma}}^{*}$ and that of the antistable ones are disjoint and both $(\widehat{A}+\widehat{B} \widehat{F})$ invariant, a further basis transformation $T_{2}$ in $\mathbb{R}^{2 n}$ can be performed so as to split the stable modes from the antistable ones. The matrix $\widehat{A}_{F, T_{2}}$ that corresponds to $\widehat{A}_{F, T_{1}}$ in this new basis has the following structure $\widehat{A}_{F, T_{2}}:=T_{2}^{-1} \widehat{A}_{F, T_{1}} T_{2}=\left[\begin{array}{ccc}M_{S} & 0 & \times \\ 0 & M_{U} & \times \\ 0 & 0 & \times\end{array}\right]$, where the eigenvalues of $M_{S}, M_{U} \in \mathbb{R}^{(n-r) \times(n-r)}$ are respectively the stable and antistable zeros of the Hamiltonian system $\widehat{\Sigma}$. Hence, the first $n-r$ columns of the product $T_{1} T_{2}$ define a basis for a controlled invariant subspace which is internally stabilizable, whose poles are the eigenvalues of $M_{S}$. Thus, this subspace is $\widehat{\mathcal{V}}_{R}$.

Lemma 5.1 Let Assumptions (A1) and (A2) hold. Let $\left[\begin{array}{l}V_{X} \\ V_{\Lambda}\end{array}\right]$ be a basis matrix of $\widehat{\mathcal{V}}_{R}$ with $V_{X}, V_{\Lambda} \in \mathbb{R}^{n \times(n-r)}$. Matrix $V_{X}$ is full rank.

Proof: By contradiction assume that $\operatorname{ker} V_{X} \neq\{0\}$ and let $N_{V_{X}}$ be a basis matrix of $\operatorname{ker} V_{X}$, then $\operatorname{im}\left[\begin{array}{c}0 \\ V_{\Lambda} N_{V_{X}}\end{array}\right] \subseteq \widehat{\mathcal{V}}_{R}$. From the controlled invariance of $\widehat{\mathcal{V}}_{R}$, one gets $\left(-C^{T} C V_{X}-A^{T} V_{\Lambda}\right) \operatorname{ker} V_{X} \subseteq V_{\Lambda} \operatorname{ker} V_{X}$, and consequently

$$
-A^{T} V_{\Lambda} \operatorname{ker} V_{X} \subseteq V_{\Lambda} \operatorname{ker} V_{X} .
$$

Since $\widehat{\mathcal{V}}_{R} \subseteq \operatorname{ker} \widehat{C}$, it ensues that $V_{\Lambda} \operatorname{ker} V_{X} \subseteq \operatorname{ker}\left(B^{T}\right)$, and from (9) the subspace $V_{\Lambda} \operatorname{ker} V_{X}$ is contained to the unobservability subspace of $\Sigma^{-T}$, which is by definition the largest $A^{T}$-invariant subspace contained in the nullspace of $B^{T}$. Moreover, modes of this invariant subspace are stable since $\widehat{\mathcal{V}}_{R}$ is an internally stable $\widehat{A}+\widehat{B} \widehat{F}$ invariant. Contradiction arises from Assumption (A1), which implies that the uncontrollability part of $\Sigma$ is stable, or equivalently that the unobservability subspace of $\Sigma^{-T}$ is antistable.

The result given in Lemma 5.1 is similar to the one found for discrete time systems in [10]. However, while in the discrete case the dimension of $\widehat{\mathcal{V}}_{R}$ equals the dimension of the state space $n$, in the continuous case such dimension is $n-r$, so that here full rankness of $V_{X}$ does not imply invertibility of $V_{X}$, since $V_{X}$ is not square in general.

In the following theorem, it is shown how the solution of the output nulling problem for $\widehat{\Sigma}$ enables the solvability condition on the initial state to be expressed in terms of the projection of the subspace $\widehat{\mathcal{V}}_{R}$ on the state-space of $\Sigma$. In other words, when the initial state is such that an optimal solution exists, a basis for the subspace of admissible initial conditions can be used to directly compute the state feedback.

Differently from the discrete case, where the optimal gain involves the inverse of $V_{X}$, here as aforementioned $V_{X}$ is not invertible, so that the Moore-Penrose pseudoinverse has to be used instead of the inverse.

Theorem 5.2 Let $\Sigma$ be left-invertible. Let Assumptions (A1) and (A2) hold. Problem 2.1 is solvable if and only if

$$
x_{0} \in i m V_{X}
$$

i.e., if and only if the initial condition $x_{0}$ belongs to the projection of $\widehat{\mathcal{V}}_{R}$ on the state-space $\mathbb{R}^{n}$ whose dimension is $n-r$. If this is the case, let $F_{X}, F_{\Lambda} \in \mathbb{R}^{m \times n}$ be obtained by partitioning a friend $\widehat{F}_{R}$ of $\widehat{\mathcal{V}}_{R}$ as $\widehat{F}_{R}=\left[\begin{array}{ll}F_{X} & F_{\Lambda}\end{array}\right]$. If condition (10) is met, the state-feedback matrix $K$ solving Problem 2.1 is given by

$$
K=-\left(F_{X}+F_{\Lambda} V_{\Lambda} V_{X}^{\dagger}\right) .
$$

Proof: Necessity and sufficiency of condition (10) can be easily proved by noting that (10) is equivalent to the existence of a costate initial condition $\lambda_{0} \in \mathbb{R}^{n}$ such that $\left[\begin{array}{l}x_{0} \\ \lambda_{0}\end{array}\right] \in \widehat{\mathcal{V}}_{R}$. The dimension of im $V_{X}$ is $n-r$ in view of Lemma 5.1. Owing to Theorem 5.1, an extended feedback matrix $\widehat{F}_{R}$ exists such that the extended trajectory obtained by applying the control $u(t)=\widehat{F}_{R}\left[\begin{array}{l}x(t) \\ \lambda(t)\end{array}\right]$ entirely lies on $\widehat{\mathcal{V}}_{R}$ and converges to the origin as $t$ approaches infinity. Hence, the projection of the extended state on $\mathbb{R}^{n}$ converges to zero as well. Hence, this is a feedback which steers the extended state along a stable trajectory evolving in $\widehat{\mathcal{V}}_{R}$. Since $x(t) \in \operatorname{im} V_{X}$, it follows that the input function can be expressed as the state feedback $u(t)=\widehat{F}_{R}\left[\begin{array}{l}x(t) \\ \lambda(t)\end{array}\right]=\left[\begin{array}{ll}F_{X} & F_{\Lambda}\end{array}\right]\left[\begin{array}{c}I_{n} \\ V_{\Lambda} V_{X}^{\dagger}\end{array}\right] x(t)$. Clearly, the dimension $\operatorname{im} V_{X}$ is $n-r$ owing to Lemma 5.1.

As is clear from the proof of Theorem 5.2, the optimal static feedback $K$ does not depend on the particular initial state satisfying (10). Moreover, under the assumption of left-invertibility of $\Sigma$ the optimal control 
$u(t)=-K x(t)$ is unique, due to the full rankness of $V_{X}$. In Section 5.1 it is shown that when the assumption of left-invertibility is removed, the optimal solution to Problem 2.1 is not unique.

We now show a simple formula for the optimal cost as a quadratic form of the initial state, which extends the very well-known result for the regular case, $[1,8]$.

Theorem 5.3 Let $x_{0} \in \mathbb{R}^{n}$ be such that (10) is satisfied. The optimal value of the cost is $J^{\star}=x_{0}^{T} V_{\Lambda} V_{X}^{\dagger} x_{0}$.

Proof: By using the Hamiltonian equations (6) we find

$$
\begin{aligned}
J^{\star} & =\int_{0}^{\infty} x^{T}(t) C^{T} C x(t) d t \\
& =\int_{0}^{\infty}\left(-x^{T}(t) \dot{\lambda}(t)-\left(\dot{x}^{T}(t)-u^{T}(t) B^{T}\right) \lambda(t)\right) d t \\
& =-\int_{0}^{\infty} \frac{d}{d t}\left(x^{T}(t) \lambda(t)\right) d t=x_{0}^{T} \lambda(0)=x_{0}^{T} V_{\Lambda} V_{X}^{\dagger} x_{0} .
\end{aligned}
$$

\subsection{Extension to nonleft-invertible systems}

In the case where system $\Sigma$ is left-invertible, given an initial state $x_{0} \in \mathbb{R}^{n}$, a solution to Problem 2.1 exists if and only if $x_{0}$ satisfies (10), and the feedback solution $u=-K x$, with $K$ defined in (11), is unique. When $\Sigma$ is not left-invertible, the optimal feedback matrix is not unique. To see this, recall that if $\Sigma$ is not left-invertible, the reachable subspace $\mathcal{R}_{\Sigma}^{*}$ on $\mathcal{V}_{\Sigma}^{*}$ differs from the origin. Recall that $\mathcal{R}_{\Sigma}^{*}$ can be interpreted as the subspace of state trajectories that can be followed indefinitely while maintaining the output function $y$ at zero. It follows that, if $u^{o}$ is optimal for Problem 2.1 and $x^{o}$ is the corresponding state trajectory, any other control leading to a state trajectory that differs from $x^{o}$ only by components on $\mathcal{R}_{\Sigma}^{*}$ leads to the same value of the cost, and is therefore optimal as well. In other words, the set of optimal controls for Problem 2.1 is parameterized modulo the inputs driving the state of $\Sigma$ on $\mathcal{R}_{\Sigma}^{*}$. Hence, when $\Sigma$ is not left-invertible, the results of Theorems 5.1 and 5.2 do not directly apply. However, the geometric approach taken here can be easily adapted to handle nonleft-invertible systems, see also [10]. Let $F$ be such that $(A+B F) \mathcal{V}_{\Sigma}^{*} \subseteq \mathcal{V}_{\Sigma}^{*}$, and such that all the eigenvalues of $(A+B F)$ restricted to $\mathcal{R}_{\Sigma}^{*}=\mathcal{V}_{\Sigma}^{*} \cap \mathcal{S}_{\Sigma}^{*}$ (which are freely assignable) are stable; let also $\Omega$ be a basis matrix of $\left(B^{-1} \mathcal{V}_{\Sigma}^{*}\right)^{\perp}$. Consider a nonleft-invertible system $\Sigma$, and the auxiliary system $\tilde{\Sigma}$ described by the triple $(A+B F, B \Omega, C)$. The system $\tilde{\Sigma}$ is left-invertible as shown in [12, Theorem 1], since the introduction of matrix $\Omega$ restricts the set of allowable inputs so as to eliminate the functions yielding motions on $\mathcal{R}_{\Sigma}^{*}$. Moreover, the invariant zeros of this new system $\tilde{\Sigma}$ are those of the original system $\Sigma$ plus those assigned through $F$, which are stable. Now, let $\tilde{K}$ be the optimal state feedback matrix for the auxiliary system. Then $K:=\Omega \tilde{K}-F$ is one of the solutions of the original problem. All the other optimal solutions can be obtained by changing $F$ within the set of stabilizing friends of $\mathcal{V}_{\Sigma}^{\star}$. This set is always non-empty when $\Sigma$ is nonleftinvertible.

\section{Concluding Remarks}

A new method for the solution of the cheap linear quadratic optimal control problem has been presented for continuous time systems. The key idea is to recast the cheap LQ regulator as an output nulling problem for the Hamiltonian system. The optimal control is sought within the class of input functions that can be expressed as a static state feedback, so as to exclude the possibility of distributions in the optimal control function. As a result of this, for certain initial conditions a solution of the cheap LQ problem may not exist. As such, a geometric analysis of independent theoretical interest on the structure and properties of the Hamiltonian system has been carried out in order to characterize the subspace of admissible initial conditions, and to determine the corresponding optimal state feedback matrix when an optimal solution does exist.

\section{References}

[1] B.D.O. Anderson and J.B. Moore. Optimal Control: Linear Quadratic Methods. Prentice Hall Int., London, 1989.

[2] G. Basile and G. Marro. Controlled and conditioned invariant subspaces in linear system theory. Journal of Optimization Theory and Applications, 3(5):306-315, May 1969.

[3] G. Basile and G. Marro. A new characterization of some structural properties of linear systems: unknown-input observability, invertibility and functional controllability. International Journal of Control, 17(5):931-943, May 1973.

[4] G. Basile and G. Marro. Controlled and Conditioned Invariants in Linear System Theory. Prentice Hall, Englewood Cliffs, New Jersey, 1992. [On-line]. Available at: http://www3.deis.unibo.it/Staff/FullProf/GiovanniMarro/ downloads.htm

[5] B.A. Francis. The optimal linear quadratic time invariant regulator with cheap control. IEEE Transactions on Automatic Control, AC-24(4):616-621, 1979.

[6] M.L.J. Hautus and L.M. Silverman. System structure and singular control. Linear Algebra and Its Applications, 50:369402, 1983.

[7] V. Ionescu, C. Oară, and M. Weiss. Generalized Riccati theory and robust control, a Popov function approach. Wiley, 1999.

[8] F.L. Lewis and V. Syrmos. Optimal Control. John Wiley \& Sons, New York, 1995.

[9] O.L. Mangasarian. Sufficient conditions for the optimal control of nonlinear systems. SIAM Journal on Control, 4(1):139-152, 1966 
[10] G. Marro, D. Prattichizzo, and E. Zattoni. A geometric insight into the discrete time cheap and singular LQR problems. IEEE Transactions on Automatic Control, 47(1):102-107, 2002.

[11] A.S. Morse. Structural invariants of linear multivariable systems. SIAM J. Control, 11(3):446-465, August 1973.

[12] L. Ntogramatzidis, D. Prattichizzo. Squaring Down LTI Systems: A Geometric Approach. Systems 83 Control Letters, 56(3):236-244, 2007.

[13] A. Saberi and P. Sannuti. Cheap and singular controls for linear quadratic regulators. IEEE Transactions on Automatic Control, AC-32(3):208-219, March 1987.

[14] A. Saberi, P. Sannuti, and B.M. Chen. $\mathrm{H}_{2}$ Optimal Control. System and Control Engineering. Prentice Hall International, London, 1995.

[15] A.A. Stoorvogel. The singular $H_{2}$ control problem. Automatica, 28(3):627-631, 1992.

[16] A.A. Stoorvogel. The $H_{\infty}$ control problem. Prentice Hall, New York, 1992.

[17] A.A. Stoorvogel and A. Saberi. The discrete-time algebraic Riccati equation and linear matrix inequality. Linear Algebra and its Applications, 274:317-365, 1998.

[18] H.L. Trentelman, A.A. Stoorvogel, and M. Hautus. Control theory for linear systems. Communications and Control Engineering. Springer, Great Britain, 2001.

[19] J.C. Willems, A. Kitapçi, and L.M. Silverman. Singular optimal control: a geometric approach. SIAM Journal of Control and Optimization, 24(2):323-337, March 1986.

[20] W.M. Wonham. Linear Multivariable Control: A Geometric Approach. Springer-Verlag, New York, 3 edition, 1985.

[21] K. Zhou, J. Doyle, and K. Glover. Robust and Optimal Control. Prentice Hall, New York, 1996. 